\title{
ON LINEAR AND NONLINEAR PERTURBATIONS OF LINEAR SYSTEMS OF ORDINARY DIFFERENTIAL EQUATIONS WITH CONSTANT COEFFICIENTS
}

\author{
BY \\ PHILIP HARTMAN AND AUREL WINTNER \\ INTRODUCTION
}

Let $J$ be a constant $d$ by $d$ matrix, let $y^{1}, \cdots, y^{d}$ be the components of a column vector $y$, and let $y^{\prime}=d y / d t$, where $t$ is a real variable. Consider the system of linear differential equations

$$
y^{\prime}=J y
$$

(with constant coefficients) and the perturbed system

$$
y^{\prime}=(J+G(t)) y,
$$

where $G(t)$ is a continuous $d$ by $d$ matrix on $0 \leqq t<\infty$. It has been shown by Perron (cf. $[9 ; 10])$ that if

$$
G(t) \rightarrow 0 \text { as } t \rightarrow \infty
$$

and if $\lambda_{1}, \cdots, \lambda_{d}$ are the eigenvalues of $J$, then (2) has $d$ linearly independent solutions $y=y_{1}(t), \cdots, y_{d}(t)$ such that $y=y_{j}(t)$ satisfies, as $t \rightarrow \infty$,

$$
\log |y(t)|=(\mu+o(1)) t,
$$

where $\mu=\operatorname{Re} \lambda_{j}$; for a sharper result, cf. [5]. It is known that if (3) is altered to the condition that each element of $t^{h-1} G(t)$ is of class $L(0, \infty)$ for a suitable number $h$, depending on $J$, then (4) can be improved to an asymptotic formula for each component of $y(t)$; cf., e.g., [5]. It is natural to ask for theorems with assertions stronger than (4), but not as strong as an asymptotic formula for the components of $y(t)$; for example, with an assertion of the type

$$
\log |y(t)|=\mu t+(l+o(1)) \log t,
$$

where $l$ is an integer, $0 \leqq l<h_{*}$, and $h_{*}$ is the maximum of the exponents $h$ in the elementary divisors $\left(\lambda-\lambda_{k}\right)^{h}$ for which $\operatorname{Re} \lambda_{k}=\mu$.

Part I will deal with such theorems for general linear systems (2), in which there is no restriction on the constant matrix $J$. Part II is concerned with $O(1)$-variants of results of this type for the case when $J$ has only one elementary divisor (of multiplicity $d$ ). It will be clear that corresponding results can be obtained for the case of an arbitrary $J$. A specialization ( $\$ 6)$ of the results to the case when (2) is equivalent to a scalar differential equation of $d$ th order,

Received by the editors March 31, 1955. 


$$
x^{(d)}+f_{1}(t) x^{(d-1)}+\cdots+f_{d-1}(t) x^{\prime}+f_{d}(t) x=0,
$$

gives theorems related to results of A. Kneser (for the case $d=2, f_{1}(t) \equiv 0$ and $f_{2}(t)$ is real-valued) and theorems related to a result of de la Vallée Poussin (for the case $d$ is arbitrary, $f_{1}, \cdots, f_{d}$ are real-valued and $t$ is restricted to a bounded interval).

Part III deals with analogues of ti.e results of Parts I and II for the case when the linear system (2) is replaced by a nonlinear system

$$
y^{\prime}=J y+F(t, y),
$$

in which $F=\left(F^{1}, \cdots, F^{d}\right)$ is a continuous vector defined for large $t$ and all $y$.

In the Appendix, there will be considered the conditions of Lonn [8] assuring that all solutions of the real, singular, differential equation

$$
d y / d x=\left(-y+x+F^{2}(x, y)\right) /\left(-x+F^{1}(x, y)\right),
$$

in a sufficiently small circle $x^{2}+y^{2} \leqq \epsilon^{2}$, are tangent to the $y$-axis or that all solutions are spirals. Lonn's sufficient condition for spirals is considerably improved (and both of Lonn's results are extended so as to apply nonconservative systems

$$
x^{\prime}=-x+F^{1}(t, x, y), \quad y^{\prime}=-y+x+F^{2}(t, x, y)
$$

as well). The final results are deduced from criteria of Kneser assuring that $x^{\prime \prime}+f(t) x=0$ is oscillatory or is nonoscillatory.

In what follows, the independent variable $t$ is real. The given functions and the solutions of the differential equations involved, unless otherwise specified, are complex-valued. It will be clear that analogues of the results can be obtained when all functions are restricted to be real-valued.

\section{Part I. The general linear case}

1. $o$ (1)-theorems. Theorem (ii) below dealing with solutions of (2) will have the following corollary involving asymptotic relations of the form (5):

(i) Let $J$ be a constant d by d matrix and let $(\lambda-\lambda(1))^{h(1)}, \cdots,(\lambda-\lambda(g))^{h(o)}$, where $h(1)+\cdots+h(g)=d$, be the elementary divisors of $J$. Let $G(t)$ be a continuous $d$ by $d$ matrix on $0 \leqq t<\infty$ satisfying, as $t \rightarrow \infty$,

$$
t^{\star} G(t) \rightarrow 0,
$$

where $\kappa=\max (h(1), \cdots, h(g))$. Then (2) has d linearly independent solutions $y=y_{j l}(t)$, where $j=1, \cdots, g$ and $l=0,1, \cdots, h(g)-1$, such that $y=y_{j l}(t)$ satisfies (5) with $\mu=\mu(j)$.

If (6) holds with a number $\kappa>h_{*}$, where $h_{*} \geqq h(j)$ for those $j$ for which $\operatorname{Re} \lambda(j)=\mu$, then $t^{h_{*}-1} G(t)$ is of class $L(0, \infty)$ and $(* *)$ in [5] provides an asymptotic formula for the components of a solution $y=y(t)$ of (2) satisfying (4).

In order to obtain finer results, it will be supposed that $J$ is in a Jordan 
normal form. This is no loss of generality since the elements of $J, G(t)$ and the components of $y(t)$ are allowed to be complex-valued. Suppose that (1) can be written as

$$
\begin{aligned}
& y^{j 1^{\prime}}=\lambda y^{j 1}, \\
& y^{j k^{\prime}}=\lambda y^{j k}+y^{j k-1}, \quad k=2, \cdots, h(j),
\end{aligned}
$$

where $\lambda=\lambda(j)=$ const.; $j=1, \cdots, g ; h(1)+\cdots+h(g)=d, \quad y^{m}=y^{j k}$ if $m=h(1)+\cdots+h(j-1)+k$ for $k=1, \cdots, h(j)$. The equations $\left(7_{j k}\right), k>1$, are missing if $h(j)=1$. Correspondingly, if the element $g_{m \gamma}(t)$ of $G(t)$ is written as $g_{m \gamma}=g_{j k \alpha \beta}$ if

$$
m=h(1)+\cdots+h(j-1)+k, \quad \gamma=h(1)+\cdots+h(\alpha-1)+\beta
$$

for $k=1, \cdots, h(j)$ and $\beta=1, \cdots, h(\alpha)$ and the summation convention is used for indices $\alpha, \beta$, then (2) can be written as

$$
\begin{aligned}
& y^{j 1^{\prime}}=\lambda y^{j 1}+g_{j 1 \alpha \beta} y^{\alpha \beta}, \\
& y^{j k^{\prime}}=\lambda y^{j k}+y^{j k-1}+g_{j k \alpha \beta} y^{\alpha \beta}, \quad k=2, \cdots, h(j) .
\end{aligned}
$$

It can be supposed that the $g$ elementary divisors of $J$ are numbered so that

$$
\mu(1) \leqq \cdots \leqq \mu(g), \text { where } \mu(j)=\operatorname{Re} \lambda(j) .
$$

Let the distinct numbers in the set (9) be denoted by $\mu^{1}<\mu^{2}<\cdots<\mu^{f}$. For a given integer $m$, where $1 \leqq m \leqq f$, an integer $j$ on the range $1 \leqq j \leqq g$ will be denoted by $p, q$ or $r$ according as $\mu(j)<\mu^{m}, \mu(j)=\mu^{m}$ or $\mu(j)>\mu^{m}$ :

$$
\mu(j)<,=\text { or }>\mu^{m} \text { according as } j=p, q \text { or } r .
$$

Let $h_{*}$ be the maximum length of the "blocks" of $J$ for which $\mu(j)=\mu^{m}$,

$$
h_{*}=\max _{q} h(q) \text {. }
$$

With reference to a particular value $q_{0}$ of $q$ and an integer $k=k_{0}$ on the range $1 \leqq k \leqq h(q)$, define integers $j_{0}, b$ by

$$
\begin{aligned}
j_{0} & =h\left(q_{0}\right)-k_{0} \\
b & =h_{*}-j_{0}
\end{aligned}
$$$$
\text { (so that } 0 \leqq j_{0} \leqq h\left(q_{0}\right)-1 \text { ), }
$$$$
\text { (so that } 1 \leqq k_{0} \leqq b \leqq h_{*} \text { ), }
$$

and let the integers $b(q)$ be defined by

$$
b(q)=k_{0} \text { or } b \text { according as } q=q_{0} \text { or } q \neq q_{0} \text {. }
$$

For $n=0,1, \cdots, j_{0}$, put

$$
D_{n}=\left\{\sum_{(n)}\left|y^{q b(q)+n}\right| 2\right\}^{1 / 2}, \text { where } \sum_{(n)}=\sum_{h(q) \geqq b(q)+n}
$$


(so that $D_{n}^{2}$ is the sum of $\left|y^{q_{0} k_{0}+n}\right|^{2}$ and of terms $\left|y^{q b+n}\right|^{2}$ in which $q \neq q_{0}$ and $h(q) \geqq b+n)$.

Using the notation (10)-(13), a refinement of (i) can be stated as follows:

(ii) Let $G(t)$ be a continuous $d$ by $d$ matrix in $0 \leqq t<\infty$ and, with reference to a fixed $\mu=\mu^{m}$ and a fixed $q=q_{0}$, let every element $g_{j k} \alpha \beta$ of $G(t)$ satisfy, when $U V>U \rightarrow \infty$,

$$
(1+\log V)^{-1} \int_{U}^{U V}\left|g_{j k \alpha \beta}\right| t^{\epsilon(\alpha \beta)-\epsilon(j k)+(\epsilon(\alpha)-\epsilon(j))\left(h_{*}-h\left(q_{0}\right)\right)} d t \rightarrow 0,
$$

where $\epsilon(j k)$ is $k$ or 1 according as $j=q$ or $j \neq q$ and $\epsilon(j)$ is 1 or 0 according as $j=q_{0}$ or $j \neq q_{0}$. Then the system (8) has $\sum_{p} h(p)+\sum_{(0)}(h(q)-b(q)+1)$ linearly independent solutions $y=y(t)$ satisfying, as $t \rightarrow \infty$, the asymptotic relations

$$
\begin{aligned}
t^{b-1} y^{j k}(t) & =o\left(D_{0}(t)\right) & & \text { if } j=p \text { and } j=r, \\
t^{b(q)-k} y^{q k}(t) & =o\left(D_{0}(t)\right) & & \text { if } k<b(q), \\
D_{n}(t) & \sim t^{n} D_{0}(t) / n ! & & \text { if } n=1, \cdots, j_{0}, \\
\log D_{0}(t) & =\mu t+o(\log t) . & &
\end{aligned}
$$

A condition sufficient for (14) is

$$
t^{h *} G(t) \rightarrow 0 \text { as } t \rightarrow \infty
$$

or, more generally,

$$
(1+\log V)^{-1} \int_{U}^{U V} t^{h_{*}-1}|G(t)| d t \rightarrow 0 \text { as } \quad U V>U \rightarrow \infty,
$$

where $|G(t)|$ is the norm of $G(t)$ (that is, $\max |G(t) y|$ for $|y|=1$ ). The asymptotic formulae (15) imply, as $t \rightarrow \infty$,

$$
|y|=(1+O(1 / t))\left\{\sum_{q}\left|y^{q h(q)}\right|^{2}\right\}^{1 / 2},
$$

in fact,

$\left(17_{2}\right)\left\{\sum_{q}\left|y^{q h(q)-n}\right|^{2}\right\}^{1 / 2}=O(1 / t)\left\{\sum_{q}\left|y^{q h(q)-n+1}\right|^{2}\right\}^{1 / 2}, n=1, \cdots, h_{*}-1$, where the sum on the left [right] is over the set of $q$ for which $h(q) \geqq n$ $[h(q) \geqq n-1]$,

$$
\begin{array}{cl}
y^{j k}=o\left(t^{1-h_{*}}\right)\left\{\sum_{q}\left|y^{q h(q)}\right|^{2}\right\}^{1 / 2} & \text { if } j \neq q, \\
\log |y|=\mu t+\left(j_{0}+o(1)\right) \log t . &
\end{array}
$$


This fact, theorem $\left(^{*}\right)$ in $[5$, pp. 51-52], and the superposition principle lead to the following:

(i bis) If condition $\left(16_{1}\right)$ or $\left(16_{2}\right)$ is satisfied then any solution $y=y(t)$ of $(8)$, for which (4) holds, satisfies $\left(17_{1}\right)-\left(17_{3}\right)$ and the relation $\left(17_{4}\right)$, for some integer $j_{0}, 0 \leqq j_{0} \leqq h_{*}-1$.

2. Proof of (ii). It can be supposed that

$$
\lambda(q)=0 .
$$

for otherwise the change of dependent variables

$$
e^{-\lambda(q) t} y^{q k} \rightarrow y^{q k}, \quad e^{-\mu t} y^{j k} \rightarrow y^{j k}
$$

transforms (8) into a system of the same type in which $g_{j k \alpha \beta}$ is replaced by $e^{i \theta} g_{j k \alpha \beta}$, where $\theta=\theta_{j k \alpha \beta}$ is a real constant.

Consider the change of independent variables $t \rightarrow s$ defined by

$$
s=\log t
$$$$
(d s=d t / t)
$$

and the change of dependent variables

$$
y=Q z
$$

given by

$$
\begin{aligned}
& y^{q k}=t^{k-b(q)} \sum_{n=1}^{k} z^{q n} /(k-n) !, \\
& y^{j k}=t^{1-b} z^{j k}
\end{aligned}
$$$$
\text { if } j \neq q \text {, }
$$

where $b, b(q)$ are defined by (12). Then (8) is transformed into the system

$$
\dot{z}^{q k}=(b(q)-k) z^{q k}+h_{q k \alpha \beta} z^{\alpha \beta},
$$

and, if $j \neq q$,

$$
\begin{aligned}
\dot{z}^{j 1} & =(\lambda t-1-b) z^{j 1}+h_{j 1 \alpha \beta} z^{\alpha \beta} \\
\dot{z}^{j k} & =(\lambda t-1-b) z^{j k}+t z^{j k-1}+h_{j k \alpha \beta} z^{\alpha \beta}
\end{aligned}
$$

where $H=\left(h_{j k \alpha \beta}\right)$ is given by

$$
H(s)=t Q^{-1} G Q \quad\left(t=e^{s}\right),
$$

and $\dot{z}=d z / d s=t z^{\prime}$; cf. [5, pp. 64-66].

The assumption (14) implies that

$$
(1+V)^{-1} \int_{U}^{U+V}\left|h_{j k \alpha \beta}\right| d s \rightarrow 0 \text { when } U+V>U \rightarrow \infty
$$

cf. [5, pp. 67-68]. Note that the coefficient of $z^{q k}$ in the principal term of $\left(23_{1}\right)$ is 0 if and only if $k=b(q)$, and that the real part of the coefficient of 
$z^{j k}$ in $\left(23_{2}\right)$ is essentially const. $t$, where const. $\gtrless 0$ according as $j=r$ or $j=p$; cf. (10) and (18).

Thus (III) in $[5$, p. 61$]$, implies that the system $\left(23_{1}\right)-\left(23_{2}\right)$ has a unique solution $z=z(s)$ satisfying, for a sufficiently large $S$, a set of (partial) initial conditions

$$
z^{j k}(S)=z_{0}^{j k}, \quad j=p \quad \text { and } \quad j=q, k \geqq b(q),
$$

where $z_{0}^{j k}$ are arbitrary constants subject to

$$
\sum_{p} \sum_{k}\left|z_{0}^{p k}\right|^{2}+\sum_{q} \sum_{k>b(q)}\left|z_{0}^{q k}\right|^{2}<\epsilon \sum_{(0)}\left|z_{0}^{q b(q)}\right|^{2}
$$

for a sufficiently small $\epsilon>0$, and satisfying, as $s \rightarrow \infty$, the asympotic relations

$$
\begin{aligned}
\log D(s) & =o(s), \text { where } D=\left\{\sum_{(0)}\left|z^{q b(q)}\right| 2\right\}^{1 / 2}, \\
z^{j k} & =o(D(s)) \text { if }(j k) \neq(q b(q)) .
\end{aligned}
$$

By virtue of (21) and (26), it follows that, as $t=e^{\circ} \rightarrow \infty$,

$$
\begin{aligned}
D_{0}(t) & \sim D(s), \\
t^{b(q)-k} y^{q k} & =o(D(s)) \text { if } k<b(q), \\
D_{n}(t) & \sim t^{n} D(s) / n !
\end{aligned}
$$

and, by virtue of $(22)$, that

$$
t^{b-1} y^{j k}=o(D(s)) \text { if } j=p \text { and } j=r .
$$

In view of (19), (26), and (27), the assertion (ii) follows.

\section{Part II. One elementary divisor}

3. A lemma. The proof of (ii) above (or rather the proof of the theorem (III) in [5] on which (ii) depends) suggests that (ii) has $O(1)$-variants. These variants will be examined in Part II in detail when $J$ has only one elementary divisor (of multiplicity $d$ ) and so, without loss of generality, it can be assumed that the eigenvalues are 0 . In this case, (1) has the form

$$
y^{1^{\prime}}=0, y^{2^{\prime}}=y^{1}, \cdots, y^{d^{\prime}}=y^{d-1} \text {. }
$$

The $O(1)$-analogue of (III) $[5$, p. 61], which will be needed below, will first be deduced.

Let $K=K(s)$ be the diagonal (not necessarily constant) matrix of coefficients of the system

$$
\dot{z}^{1}=\lambda^{1}(s) z^{1}, \cdots, \dot{z}^{d}=\lambda^{d}(s) z^{d},
$$

which will be written as 


$$
\dot{z}=K(s) z
$$$$
\dot{z}=d z / d s
$$

and let

$$
\mu^{1}(s) \leqq \cdots \leqq \mu^{d}(s), \text { where } \mu^{j}=\operatorname{Re} \lambda^{j}
$$

Let $j_{1}, j_{2}$ denote a pair of integers satisfying $1 \leqq j_{1} \leqq j_{2} \leqq d$. An integer $j=1, \cdots, d$ will be denoted by $p, q$ or $r$ according as $j<j_{1}, j_{1} \leqq j \leqq j_{2}$ or $j_{2}<j:$

$$
j=p, q \text { or } r \text { according as } j<j_{1}, j_{1} \leqq j \leqq j_{2} \text { or } j_{2}<j \text {; }
$$

so that the set of integers $p$ and/or $r$ can be empty, but the set of integers $q$ is not. Let

$$
\mu=\min _{q} \mu^{q} \text { and } \nu=\max _{q} \mu^{q},
$$

so that $\mu=\mu^{j}$ if $j=j_{1}$ and $\nu=\mu^{j}$ if $j=j_{2}$, and let there exist a constant $c>0$ such that

$$
\mu^{j} \leqq \mu-c \text { if } j=p \text { and } \mu^{j} \geqq \nu+c \text { if } j=r \text { for large } s .
$$

Finally, let

$$
M=\sum_{p}\left|z^{p}\right|^{2}, \quad L=\sum_{q}\left|z^{q}\right|^{2}, \quad N=\sum_{r}\left|z^{r}\right|^{2}
$$

so that

$$
|z|^{2}=M+L+N \text {. }
$$

For a given number $\theta$ on the range

$$
0<\theta<1
$$

let $\delta=\delta(\theta)$ denote the number

$\left(38_{1}\right) \quad \delta=1 / 2-(1-\theta)^{1 / 2} / 2$; so that $0<\delta<1 / 2$ and $2 \delta(1-\delta)=\theta / 2$.

Let $\eta$ be a number on the range

$$
0<\eta<1 / 2-\delta
$$

and let

$$
\epsilon=2(\delta+\eta) \text {, so that } 0<\epsilon<1 \text {. }
$$

LEMMA 1. Let $K=K(s)$ be a continuous (diagonal) d by d matrix on $0 \leqq s<\infty$ and let (30) reduce to (29). Let there exist integers $j_{1}, j_{2}$, where $1 \leqq j_{1} \leqq j_{2} \leqq d$, and a constant $c>0$ such that (32)-(34) hold. Let $H=H(s)$ be a continuous $d$ by $d$ matrix on $0 \leqq s<\infty$ with a norm $|H(s)|$ satisfying, for some $\theta$ on the range (37),

$$
|H(s)| \leqq \theta c / 2 \text { for large } s
$$


or, more generally, for some $\eta$ satisfying $\left(38_{2}\right)$, let

(39 bis) $\quad \int_{U}^{U+V}|H(s)| d s<\theta c V / 2+\eta$ for large $U$ and $U+V>U$.

Finally, let $z_{0}^{q}$, where $q=j_{1}, \cdots, j_{2}$, be a set of $j_{2}-j_{1}+1$ numbers, not all 0 . Then, if $S$ is sufficiently large, the system

$$
\dot{z}=(K(s)+H(s)) z
$$

has a solution $z=z(s)$ satisfying the (partial) set of initial conditions

$$
z^{p}(S)=0 ;
$$

$$
z^{q}(S)=z_{0}^{q}
$$

and the asymptotic formulae, as $s \rightarrow \infty$,

$$
\lim \sup (M(s)+N(s)) / L(s) \leqq \epsilon /(1-\epsilon),
$$

where $\epsilon$ is given by (38), and

$$
\begin{aligned}
& \lim \inf s^{-1} \log L^{1 / 2}(s) \geqq \mu-\theta c / 2(1-\epsilon)^{1 / 2}, \\
& \lim \sup s^{-1} \log L^{1 / 2}(s) \leqq \nu+\theta c / 2(1-\epsilon)^{1 / 2} .
\end{aligned}
$$

The number $\epsilon$ in (43) and (44) can be replaced by $\epsilon / 2$ if $j_{1}=1$ or $j_{2}=d$ (that is, if the set of integers $p$ or $r$ is vacuous).

It will be clear from the proof that (41) can be replaced by $z^{p}(S)=z_{0}^{p}$, where $z_{0}^{p}$ are arbitrary numbers such that $\sum_{p}\left|z_{0}^{p}\right|^{2} / \sum_{q}\left|z_{0}^{q}\right|^{2}$ is sufficiently small.

The lemma is a variant of a theorem of Perron ([9, pp. 141-142]; cf. [5]). It is clear from Perron's result [9] (or from [5]) that Lemma 1 has an analogue for the case when $K(s)$ is not diagonal but is, for example, a triangular matrix. Lemma 1 will be applied to obtain $O(1)$-variants of (i) in the case (28) of (1). It can be used to obtain $O(1)$-variants of (1) in the general case (7) of (1), by first using the change of variables (19) and (21)(22) in (2) and then employing a device of Perron which, in this case, is another change of dependent variables $z \rightarrow w$, defined by $z^{q k}=w^{q k} z^{j k}=a^{k} w^{j k}$ if $j \neq q$ and $a>0$ is a sufficiently small number. The avoidance of the use of successive approximations in the proof of the lemma makes possible the extension of the proof to nonlinear systems (cf. Part III below), without the assumption of Lipschitz conditions as in [9].

It is clear from [11] that Lemma 1 also has an analogue if the constant $c>0$ in conditions (34) and (39) is replaced by a continuous function $c=c(s)$ $>0$ satisfying $\int^{\infty} c(s) d s=\infty$. But this generalization can be deduced from Lemma 1 by the change of independent variables $s \rightarrow t$ defined by $d t=c(s) d s$.

REMARK 1 . The solution $z=z(s)$ of (40) satisfying (43)-(44) can be chosen to be independent of $\theta$, in the sense that if ( $39 \mathrm{bis}$ ) holds for arbitrarily small 
$\theta>0$ (so that $\epsilon>0$ be chosen arbitrarily small), then (43)-(44) hold with $\theta=\epsilon=0$; cf. (III), [5, p. 61].

REMARK 2. It will follow from the proof that the assertion (43) remains valid if (39 bis) is relaxed to

$$
\int_{U}^{U+V} \max g_{k}(s) d s<\theta c V / 2+\eta \text { for } V>0 \text { and large } U, k=1,2,
$$

where $g_{k}(s)=g_{k}(s, z)$ are defined by

$$
\begin{aligned}
& g_{1}(s)=2 \operatorname{Re}\left\{(L+N) \sum_{j=p} \sum_{k=1}^{d} h_{j k} z^{k} \bar{z}^{j}-M \sum_{j=q, r} \sum_{k=1}^{d} h_{j k} z^{k} \bar{z}^{j}\right\}, \\
& g_{2}(s)=2 \operatorname{Re}\left\{N \sum_{j=p, q} \sum_{k=1}^{d} h_{j k} z^{k} \bar{z}^{j}-(L+M) \sum_{j=r} \sum_{k=1}^{d} h_{j k} z^{k} \bar{z}^{j}\right\},
\end{aligned}
$$

and where the max in $\left(45_{k}\right)$ refers to the maximum with respect to $z$ on the respective sets in the $z$-space:

$$
\begin{aligned}
|z| & =1 & \text { and } & 1-\epsilon / 2 \leqq L+N \leqq 1-\delta, \\
|z| & =1 & \text { and } & \delta \leqq N \leqq \epsilon / 2 .
\end{aligned}
$$

(In particular, the inequalities $\left(45_{k}\right)$, where the $\max$ refers to $|z|=1$, are sufficient for the assertion (43).)

It may be remarked that if $|z|=1$, then $\left|g_{k}(s)\right| \leqq|H(s)|$ even if the $\operatorname{Re}$ is omitted in $\left(46_{k}\right)$. For, by Schwarz's inequality, the absolute value of the expression $2\{\cdots\}$ in $\left(46_{1}\right)$ is majorized by

$$
2(L+N)\left(\sum_{j=p}\left|\sum_{k=1}^{d} h_{j k} z^{k}\right|^{2}\right)^{1 / 2} M^{1 / 2}+2 M\left(\sum_{j=q, r}\left|\sum_{k=1}^{d} h_{j k} z^{k}\right|^{2}\right)^{1 / 2}(L+N)^{1 / 2} .
$$

Another application of Schwarz's inequality shows that this quantity is, in turn, majorized by

$$
2(L+N)^{1 / 2} M^{1 / 2}\left\{\sum_{j=1}^{d}\left|\sum_{k=1}^{d} h_{j k} z^{k}\right|^{2}\right\}^{1 / 2}(M+L+N)^{1 / 2} .
$$

Since $2(L+N)^{1 / 2} M^{1 / 2} \leqq M+L+N=|z|^{2}$, the last expression does not exceed $|z|^{2}|H z|^{1 / 2}|z|$. Hence $\left|g_{1}(s)\right| \leqq|H(s)|$ if $|z|=1$.

Similarly, it can be shown that $\left|g_{2}(s)\right| \leqq|H(s)|$ if $|z|=1$.

REMARK 3. It will also be clear from the proof that the assertion (43) and the assumption

$$
\limsup _{V \rightarrow \infty} V^{-1} \int^{V}\left\{\sum_{j=q} \sum_{k=1}^{d}\left|h_{j k}\right|^{2}\right\}^{1 / 2} d s \leqq \theta c / 2
$$


(48 bis) $\quad \limsup _{V \rightarrow \infty} V^{-1} \int^{V} \max _{|z|=1} \operatorname{Re}\left\{\sum_{q} \sum_{k=1}^{d} h_{q k} z^{k} \bar{z}^{q}\right\}^{1 / 2} d s \leqq \theta c / 2$ assure (44).

4. Proof of Lemma 1. This will only be indicated. Since

$$
\left(\left|z^{j}\right|^{2}\right)^{\cdot}=\left(z^{j}\right)^{\cdot} \bar{z}^{j}+z^{j}\left(\bar{z}^{j}\right)^{\cdot}=2 \operatorname{Re} \dot{z}^{j} \bar{z}^{j},
$$

it follows from (31), (32), and (40) that

$$
\begin{aligned}
& \dot{L} \geqq 2 \mu L+2 \operatorname{Re} \sum_{q} \sum_{k=1}^{d} h_{q k} z^{k} \bar{z}^{q}, \\
& \dot{L} \leqq 2 \nu L+2 \operatorname{Re} \sum_{q} \sum_{k=1}^{d} h_{q k} z^{k} \bar{z}^{q}
\end{aligned}
$$

and

$$
\begin{aligned}
& \dot{M} \leqq 2(\mu-c) M+2 \operatorname{Re} \sum_{p} \sum_{k=1}^{d} h_{p q} z^{k} \bar{z}^{p}, \\
& N \geqq 2(\nu+c) N+2 \operatorname{Re} \sum_{r} \sum_{k=1}^{d} h_{r k} z^{k} \bar{z}^{r} .
\end{aligned}
$$

Corresponding to a solution $z=z(s) \not \equiv 0$ of $(40)$, the function

$$
v=v(s)=(L+N) /(M+L+N)
$$

satisfies

$$
0 \leqq v \leqq 1,
$$

is identically 1 when there are no integers $p$, and otherwise satisfies the Riccati differential inequality

$$
\dot{v} \geqq 2 c v(1-v)-2 \operatorname{Re}\{\cdots\}(M+L+N)^{-2},
$$

where $\{\cdots\}$ is the expression in $\left(46_{1}\right)$. This last fact follows from

$$
\begin{gathered}
\dot{v}=\{(L+N) \cdot M-(L+N) \dot{M})\} /(M+L+N)^{2} \text { and } \\
(L+N) M /(M+L+N)^{2}=v(1-v) .
\end{gathered}
$$

The definition of $g_{1}(s)=g_{1}(s, z)$ in (46) shows that the differential inequality in the last formula line can be written as

$$
\dot{v} \geqq 2 c v(1-v)-g_{1}(s) \text {. }
$$

Similarly, the function

$$
w=w(s)=N /(M+L+N)
$$

satisfies 


$$
0 \leqq w \leqq 1,
$$

is identically 0 when there are no integers $r$, and otherwise satisfies the Riccati differential inequality

$$
\dot{w} \geqq 2 c w(1-w)-g_{2}(s) .
$$

In view of the remarks following (47), the assumption (39 bis) implies that

$$
\int_{U}^{U+V} g_{k}(s, z(s)) d s<\theta c V / 2+\eta
$$

if $U$ is sufficiently large, $U+V>U$ and, according as $k=1$ or $k=2$,

$$
(\delta \leqq) 1-\epsilon / 2 \leqq v \leqq 1-\delta \quad \text { or } \quad\left(58_{2}\right) \quad \delta \leqq w \leqq \epsilon / 2(\leqq 1-\delta)
$$

holds on $U \leqq s \leqq U+V$. Since $(1-\delta)-(1-\epsilon / 2)=\eta$ and $\epsilon / 2-\delta=\eta$ and since $\left(38_{1}\right)$ and $\left(58_{1}\right),\left(58_{2}\right)$ imply that $2 c v(1-v) \geqq \theta c / 2,2 c w(1-w) \geqq \theta c / 2$, it follows that if

$$
v\left(s_{0}\right) \geqq 1-\delta \quad \text { or }
$$

$$
w\left(s_{0}\right) \geqq \epsilon / 2
$$

for some sufficiently large $s=s_{0}$, then

$$
v(s) \geqq 1-\epsilon / 2 \quad \text { or }
$$

$$
w(s) \geqq \delta
$$

for $s \geqq s_{0}$; cf. the proof of the lemma $[6, \S 3]$.

An adaptation of the proof of $\left({ }^{*}\right)$ in [5] (in which the assertions involving (59)-(60) play the role of Lemma 2 bis $[5$, p. 54]) shows that (40) has a solution $z=z(s)$ satisfying (41)-(42), for a sufficiently large $S$, and that

$$
v(s) \geqq 1-\epsilon / 2, \quad w(s) \leqq \epsilon / 2 \quad(S \leqq s<\infty) .
$$

In view of (51) and (54), this implies that $(L+N) \geqq(1-\epsilon / 2)(L+N+M)$ and $N \leqq(\epsilon / 2)(L+N+M)$; so that $L \geqq(1-\epsilon)(L+N+M)$, that is,

$$
N+M \leqq \epsilon L /(1-\epsilon) \quad(S \leqq s<\infty) .
$$

This proves (43). Note that if $N \equiv 0$ or $M \equiv 0$, then $\epsilon$ can be replaced by $\epsilon / 2$ in (62), hence in (43).

By $\left(49_{1}\right)$ and Schwarz's inequality,

$$
\dot{L} \geqq 2 \mu L-2|H||z| L^{1 / 2} .
$$

Since, by (62), $|z|=(L+N+M)^{1 / 2} \leqq L^{1 / 2} /(1-\epsilon)^{1 / 2}$,

$$
\dot{L} \geqq\left(2 \mu-2|H| /(1-\epsilon)^{1 / 2}\right) L,
$$

the relation $\left(44_{1}\right)$ follows from (39 bis). The relation $\left(44_{2}\right)$ can be obtained similarly.

5. The system (2), when (1) is (28). Before applying Lemma 1 to the situation at hand, it can be noted that (ii) implies the following: 
(I) Let (1) be the system (28). Let $G=G(t)$ be a continuous $d$ by $d$ matrix on $0 \leqq t<\infty$ satisfying

$$
t^{d} G(t) \rightarrow 0 \quad \text { as } \quad t \rightarrow \infty
$$

or, more generally,

(63 bis) $\quad(1+\log V)^{-1} \int_{U}^{U V} t^{d-1}|G(t)| d t \rightarrow 0$ when $\quad U V>U \rightarrow \infty$.

Then, for $m=1, \cdots, d$, the system (2) has a solution $y=y(t)$ the components of which satisfy, as $t \rightarrow \infty$,

$$
\begin{aligned}
\log \left|y^{m}(t)\right| & =o(\log t), \\
y^{j}(t) & =o\left(t^{j-m}|y(t)|\right) \quad \text { if } j<m, \\
y^{j}(t) & \sim t^{j-m} y(t) /(j-m) ! \text { if } j>m .
\end{aligned}
$$

In order to obtain an $O(1)$-variant and refinement of this result, by the use of Lemma 1, introduce the following notations: Let $Q=Q(t)$ denote the triangular matrix of the affine transformation

$$
y=Q z, \quad \text { where } \quad y^{j}=t^{j-1} \sum_{k=1}^{j} z^{k} /(j-k) !
$$

and let

$$
H=t Q^{-1} G Q
$$

In terms of the matrix $H=\left(h_{j k}\right)$, an integer $m(1 \leqq m \leqq d)$, and a number $\theta(0<\theta<1)$, put

$\left(67_{1}\right) \quad g_{1}(t)=\max 2 \operatorname{Re}\left\{\sum_{n=1}^{m-1}\left|z^{n}\right|^{2} \sum_{j=m}^{d} \sum_{k=1}^{d} h_{j k} z^{k} \bar{z}^{j}-\left.\sum_{n=m}^{d}\left|z^{n}\right|\right|^{2} \sum_{j=1}^{m-1} \sum_{k=1}^{d} h_{j k} z^{k} \bar{z}^{j}\right\}$,

$$
\begin{aligned}
g_{2}(t)=\max 2 \operatorname{Re} & \left\{\sum_{n=1}^{m}\left|z^{n}\right|^{2} \sum_{j=m+1}^{d} \sum_{k=1}^{d} h_{j k} z^{k} \bar{z}^{j}\right. \\
& \left.-\sum_{n=m+1}^{d}\left|z^{n}\right|^{2} \sum_{j=1}^{m} \sum_{k=1}^{d} h_{j k} z^{k} \bar{z}^{j}\right\},
\end{aligned}
$$

where the max refers to a maximum with respect to $z$ on the respective domains

$$
\begin{array}{r}
|z|=1 \text { and } 1-\epsilon / 2 \leqq \sum_{j=m}^{d}\left|z^{j}\right|^{2} \leqq 1-\delta, \\
|z|=1 \text { and } \delta \leqq \sum_{j=m+1}^{d}\left|z^{j}\right|^{2} \leqq \epsilon / 2
\end{array}
$$


and $\epsilon, \delta$ are defined, in terms of $\theta$, by (38).

$\left(I^{*}\right)$ Let (1) be given by (28). Let $m$ be an integer on the range $1 \leqq m \leqq d$. Let $\theta>0$ be such that the number $\delta=\delta(\theta)$ in $\left(38_{1}\right)$ satisfies

$$
0<2 \delta(\theta)<\left(1+\alpha_{m}^{2}\right)^{-1} \text {, where } \alpha_{j}^{2}=\sum_{n=1}^{j-1}(1 / n !)^{2} .
$$

Let $G=G(t)$ be a continuous $d$ by $d$ matrix with the property that the functions $g_{k}(t)$, defined by (66)-(68) for the given $m$ and $\theta$, satisfy

$$
g_{k}(t) \leqq \theta / 2 \text { for large } t
$$$$
(k=1,2)
$$

or, more generally, let there exist an $\eta>0$ with the properties that $\left(38_{2}\right)$ holds and that $\left(3_{3}\right)$ satisfies

$$
0<\epsilon<\left(1+\alpha_{m}^{2}\right)^{-1}
$$

and

$$
\int_{U}^{U V} t^{-1} g_{k}(t) d t<\theta \log V / 2+\eta \text { for large } U \text { and } U V>U .
$$

Then (1) has a solution $y=y(t)$ satisfying, as $t \rightarrow \infty$,

$$
\lim \sup t^{m-i}\left|y^{i} / y^{m}\right| \leqq \epsilon^{*} \alpha_{j+1},
$$$$
j<m \text {, }
$$
$\lim \sup \left|(j-m) ! t^{m-i} y^{j} / y^{m}-1\right| \leqq \epsilon^{*}\left(\alpha_{j+1}(j-m) !+\alpha_{m}\right), \quad i>m$, where

$$
\epsilon^{*}=\epsilon^{1 / 2} /\left((1-\epsilon)^{1 / 2}-\epsilon^{1 / 2} \alpha_{m}\right) .
$$

If, in addition, it is assumed that, as $V \rightarrow \infty$,

$$
\lim \sup (\log V)^{-1} \int^{V} t^{-1}|H(t)| d t \leqq c / 2, \quad \quad 0 \leqq c \leqq 1,
$$

then

$$
\lim \sup |\log | y^{m}(t)|/ \log t| \leqq c / 2(1 \quad \epsilon)^{1 / 2} .
$$

REMARK. The inequality $\left(71_{1}\right)$ is equivalent to

$$
0<\epsilon \alpha_{m}^{2} /(1-\epsilon)<1 .
$$

If $m=1$, then $\alpha_{m}=0$ and (69) is equivalent to $0<\theta<1$. In the case $m=d>1$, conditions (69), $\left(71_{1}\right)$ can be replaced by

(69 bis) $0<\delta(\theta)<\left(1+\alpha_{d}^{2}\right)-1, \quad\left(71_{1}\right.$ bis $) \quad 0<\epsilon / 2<\left(1+\alpha_{d}^{2}\right)^{-1}$, respectively. In both of these cases $(m=1, m=d>1), \epsilon$ can be replaced by $\epsilon / 2$ in (73) and (75). This will be clear from the proof and the last part of Lemma 1. 
In view of the remarks following (47), the functions $g_{k}(t)$ satisfy $\left|g_{k}(t)\right|$ $\leqq H(t) \mid$. It is readily verified from (65) that

$$
Q(t)=\left(\begin{array}{lllll}
1 & & & \\
& t & & \\
& & \ddots & \\
& & \cdot & t^{d-1}
\end{array}\right)\left(\begin{array}{llll}
1 & & & \\
1 / 1 ! & 1 & \\
\vdots & & \vdots & \cdot \\
1 /(d-1) ! & 1 /(d-2) ! \cdots & 1
\end{array}\right),
$$

where the first matrix on the right is diagonal and the second is $e^{J}$ (cf. [5, pp. 65-66]); so that, if $G=\left(g_{j k}\right)$,

$$
h_{j k}=t \sum_{m=1}^{j} \sum_{n=k}^{d}(-1)^{i-m} t^{n-m} g_{m n} /(j-m) !(n-k) !
$$

Since $n-m \leqq d-1$, it follows that, as $t \rightarrow \infty$,

$$
|H(t)|=O\left(t^{d}|G(t)|\right) \text {. }
$$

This makes it clear that if (63 bis) is satisfied, then $\left(71_{2}\right)$ holds for arbitrarily small $\theta>0$. Hence (I) is contained in $\left(I^{*}\right)$; cf. Remark 1 following the statement of Lemma 1.

In order to prove $\left(I^{*}\right)$, make the variation of constants (65) in (2) and the change of independent variables $t \rightarrow s=\log t$. Then, since (1) is given by (28), the system (2) becomes

$$
\dot{z}=(K+H) z \text {, where } \dot{z}=d z / d s \text { and } d s=d t / t \text {, }
$$

$H$ is given by (66) (cf. (77)) and $K$ is the diagonal matrix with the diagonal elements $\lambda^{1}=0, \lambda^{2}=-1, \cdots, \lambda^{d}=1-d$. (Note that the inequalities corresponding to (31) are reversed, which explains an apparent discrepancy in (67) and (46).)

In order to apply Lemma 1 , choose $j_{1}=j_{2}=m$; so that $\mu=\nu=1-m$,

$$
M=\sum_{k<m}\left|z^{k}\right|^{2}, \quad L=\left|z^{m}\right|^{2}, \quad N=\sum_{k>m}\left|z^{k}\right|^{2},
$$

and $c$ can be chosen to be 1 . Since $d s=d t / t$ (and $s=\log t$ ), it follows from Lemma 1 and the remarks concerning (46), that (71) implies for (79) the existence of a solution $z=z(s)$ with components satisfying

$$
\sum_{j \neq m}\left|z^{j}\right|^{2} \leqq \epsilon\left|z^{m}\right|^{2} /(1-\epsilon) \text { for large } t
$$

(or large $s=\log t$ ) (cf. (43)) and that (80) and (74) imply that

$$
\limsup _{i \rightarrow \infty}|\log | z^{m}(t)|/ \log t-(1-m)| \leqq c / 2(1-\epsilon)^{1 / 2} .
$$

By (65), the definition of $\alpha_{j}$ in (69), and Schwarz's inequality, the solu- 
tion $y=y(t)$ of (1) corresponding to $z=z(s)$ satisfies

$$
\left|t^{1-j} y^{j}\right| \leqq\left(\sum_{k=1}^{j}\left|z^{k}\right|^{2}\right)^{1 / 2} \alpha_{j+1}
$$

Hence, by (80),

$$
\left|t^{1-i} y^{j}\right| \leqq \epsilon^{1 / 2} \alpha_{j+1}\left|z^{m}\right| /(1-\epsilon)^{1 / 2} \text { if } j<m .
$$

Similarly,

$$
\left|\cdot t^{1-m} y^{m}-z^{m}\right| \leqq \epsilon^{1 / 2} \alpha_{m}\left|z^{m}\right| /(1-\epsilon)^{1 / 2}
$$

and

$$
\left|t^{1-j} y^{j}-z^{m} /(j-m) !\right| \leqq \epsilon^{1 / 2} \alpha_{j+1}\left|z^{m}\right| /(1-\epsilon)^{1 / 2} \text { if } j>m .
$$

The inequalities (76) and (82) imply that

$$
\left|z^{m}\right| \leqq t^{1-m}\left|y^{m}\right| \epsilon^{*}(1-\epsilon)^{1 / 2} / \epsilon^{1 / 2},
$$

by virtue of (73). The relations (72) follow from $\left(82_{1}\right),\left(82_{3}\right)$, and $\left(82_{4}\right)$. Finally, (75) follows from (81) and $\left(82_{2}\right)$. This proves $\left(I^{*}\right)$.

6. An equation of $d$ th order. Consider a linear differential equation of $d$ th order,

$$
x^{(d)}+f_{1}(t) x^{(d-1)}+\cdots+f_{d-1}(t) x^{\prime}+f_{d}(t) x=0, \quad d>1,
$$

for a scalar function $x$, where $f_{j}(t)$ is continuous for $0 \leqq t<\infty$ and $j=1, \cdots, d$. On defining $y=\left(y^{1}, \cdots, y^{d}\right)$ by

$$
y^{d}=x, \quad y^{d-1}=x^{\prime}, \cdots, y^{1}=x^{(d-1)},
$$

the equation (83) becomes a system (2) for $y=\left(y^{1}, \ldots, y^{d}\right)$ in which (1) is (28) and $G(t)$ is the matrix in which the first row is

$$
\left(-f_{1},-f_{2}, \cdots,-f_{d}\right)
$$

and in which the other elements are 0 . Correspondingly, the first row of the matrix $H(t)$ in (66) is

$$
\left(-\sum_{j=1}^{d} f_{j} t^{i} /(j-1) !,-\sum_{j=2}^{d} f_{j} t^{i} /(j-2) !, \cdots,-t^{d} f_{d}\right)
$$

and the $j$ th row is $(-1)^{j+1}$ times the first row; cf. (77). Hence (67) becomes $\left(85_{1}\right) g_{1}(t)=\max 2 \operatorname{Re} g(t ; z)\left\{\sum_{n=1}^{m-1}\left|z^{n}\right|^{2} \sum_{j=m}^{d}(-1)^{j} \bar{z}^{j}-\sum_{n=m}^{d}\left|z^{n}\right|^{2} \sum_{j=1}^{m-1}(-1)^{i} \bar{z}^{i}\right\}$, $\left(85_{2}\right) g_{2}(t)=\max 2 \operatorname{Re} g(t ; z)\left\{\sum_{n=1}^{m}\left|z^{n}\right|^{2} \sum_{j=m+1}^{d}(-1)^{i} \bar{z}^{j}-\sum_{n=m+1}^{d}\left|z^{n}\right|^{2} \sum_{j=1}^{m}(-1)^{i} \bar{z}^{j}\right\}$, where 


$$
g(t, z)=\sum_{k=1}^{d} \sum_{n=k}^{d} f_{n} t^{n} z^{k} /(n-k) !
$$

and the max in $\left(85_{1}\right),\left(85_{2}\right)$ refers to the maximum with respect to $z=\left(z^{1}, \cdots\right.$, $\left.z^{d}\right)$ on the respective sets $\left(68_{1}\right),\left(68_{2}\right)$.

Thus, (I) has the following corollary:

(II) In (83), let $f_{1}(t), \cdots, f_{d}(t)$ be continuous functions on $0 \leqq t<\infty$ satisfying

$$
\left|t f_{1}\right|+\cdots+\left|t^{d} f_{d}\right| \rightarrow 0 \text { as } t \rightarrow \infty
$$

or, more generally,

$$
(1+\log V)^{-1} \int_{U}^{U V}\left(\left|f_{1}\right|+\left|t f_{2}\right|+\cdots+\left|t^{d-1} f_{d}\right|\right) d t \rightarrow 0
$$

when $U V>U \rightarrow \infty$.

Then, for $m=0,1, \cdots, d-1$, the differential equation (83) has a solution $x=x(t)$ the derivatives of which satisfy

$$
\begin{aligned}
\log \left|x^{(m)}(t)\right| & =o(\log t), & & \\
x^{(j)}(t) & =o\left(t^{m-i}\left|x^{(m)}(t)\right|\right) & & \text { if } j=m+1, \cdots, d, \\
x^{(j)}(t) & \sim t^{m-j} x^{(m)}(t) & & \text { if } j=0,1, \cdots, m-1 .
\end{aligned}
$$

It is understood that $(87)$ implies that $x^{(m)}(t)$ and, therefore, $x^{(m-1)}, \cdots$, $x^{\prime}, x$ do not vanish for large $t$. A similar remark applies to (88) below.

(I*) implies the following $O(1)$-variant of (II):

(II $\left.{ }^{*}\right)$ Let $f_{1}(t), \cdots, f_{d}(t)$ be continuous for $0 \leqq t<\infty$. Let $m$ be an integer satisfying $0 \leqq m \leqq d-1$ and let $\theta$ be a number satisfying (69) when $m$ is replaced by $d-m$. Let the functions (85), in which $m$ is replaced by $d-m$, satisfy (71). Then (83) has a solution $x=x(t)$ the derivatives of which satisfy, as $t \rightarrow \infty$,

$$
\lim \sup t^{i-m}\left|x^{(j)} / x^{(m)}\right| \leqq \epsilon^{*} \alpha_{d-j-1}, \quad d>j>m
$$

$\left(88_{2}\right) \quad \lim \sup \left|(m-j) ! t^{j-m} x^{(j)} / x^{(m)}-1\right| \leqq \epsilon^{*}\left(\alpha_{d-j-1}(m-j) !+\alpha_{d-m}\right)$,

$$
0 \leqq j<m \text {, }
$$

where $\epsilon^{*}$ is defined by (73) in which $m$ is replaced by $d-m$. In addition, if, as $V \rightarrow \infty$,

(89) $\lim \sup (\log V)^{-1} \int^{V}\left\{\sum_{j=1}^{d}\left|\sum_{n=j}^{d} f_{n} t^{n-1} /(n-j) !\right|^{2}\right\}^{1 / 2} d t \leqq c / 2,0 \leqq c \leqq 1$, then, as $t \rightarrow \infty$,

$$
\lim \sup |\log | x^{(m)}|/ \log t| \leqq c / 2(1-\epsilon)^{1 / 2} .
$$

Cf. the remark following the statement of $\left(I^{*}\right)$ which becomes applicable to the cases $m=0, d-1>0$ of $\left(\mathrm{II}^{*}\right)$. 
When $d=2$, then $g_{1}(t) \equiv 0$ or $g_{2}(t) \equiv 0$ in (85) according as $m=1$ or $m=2$. If (83) is of the form

$$
x^{\prime \prime}+f(t) x=0
$$

(so that $f_{1}(t) \equiv 0$ and $\left.f_{2}(t) \equiv f(t)\right)$, then, according as $m=1$ or $m=2$, the function $g_{2}(t)$ or $g_{1}(t)$ is a maximum of the function $2 \operatorname{Re} t^{2} f(t)\left(z^{1}+z^{2}\right)^{2} \bar{z}^{1} \bar{z}^{2}$, over a suitable domain in the $\left(z^{1}, z^{2}\right)$-space. Note that $\left|\left(z^{1}+z^{2}\right)^{2} z^{1} z^{2}\right| \leqq\left(\left|z^{1}\right|^{2}\right.$ $\left.+\left|z^{2}\right|^{2}\right)^{2}$. When $m=1$ (and so, $d-m=1$ ), the condition (69) is merely to the effect that $0<\theta<1$; when $m=0$ (so that $d-m=2$ ), the condition (69 bis) is also equivalent to $0<\theta<1$. Thus the conditions of $\left(\mathrm{II}^{*}\right)$ for the case (91) of (83), with $d=2$ and $m=0$ or $m=1$, are satisfied if $4 t^{2}|f(t)| \leqq \theta<1$. This makes it clear that $\left(\mathrm{II}^{*}\right)$ can be considered as a generalization of a theorem of A. Kneser which asserts that if $f(t)$ is real-valued and satisfies $4 t^{2} f(t) \leqq 1$ for large $t$, then (91) is nonoscillatory; cf. the Appendix.

Assertion (II*) implies more than the mere fact that (91) is nonoscillatory when $4 t^{2}|f(t)| \leqq \theta<1$ for large $t$. Under this condition, (91) has a pair of linearly independent solutions which vanish for large $t$ and satisfy, as $t \rightarrow \infty$,

$$
\text { lim sup }\left|t x^{\prime} / x-1\right| \leqq \gamma(\theta) \text { and } \lim \sup \left|t x^{\prime} / x\right| \leqq \gamma(\theta),
$$

respectively, where $\gamma(\theta)$ is a constant (which satisfies $\gamma(\theta) \rightarrow 0$ as $\theta \rightarrow 0$ ); cf. (88). When $f(t)$ is real-valued and $4 t^{2}|f(t)| \leqq \theta<1$, it can be shown that $\gamma(\theta)$ can be chosen to be the number $\delta(\theta)(<1)$ in $\left(38_{1}\right)$; cf. [1, p. 570] and [3, p. 722].

For arbitrary $d$, the conditions (69)-(70) of (II*) are satisfied for every $m=1,2, \cdots, d$ if $\left(38_{1}\right)$ satisfies (69) for $m=d$ (that is, if

$$
\left\{1-(1-\theta)^{1 / 2}\right\}\left\{1+\left(\sum_{n=1}^{d-1}(1 / n !)^{2}\right)\right\}<1
$$

holds) and if

$$
\left\{d \sum_{j=1}^{d}\left|\sum_{n=j}^{d} f_{n}(t) t^{n} /(n-j) !\right|^{2}\right\}^{1 / 2} \leqq \theta / 2 \text { for large } t .
$$

In fact, the expression on the left of the last inequality is

$$
\left\{\sum_{j=1}^{d} \sum_{k=1}^{d}\left|h_{j k}\right| 2\right\}^{1 / 2},
$$

which is a majorant for the norm $|H(t)|$ of the corresponding matrix (66); cf. the remarks following (84). In particular, (92) and (93) assure that no solution $x=x(t) \not \equiv 0$ of $(83)$ has infinitely many zeros on $0 \leqq t<\infty$.

It can be remarked that there seems to be some connection between the criterion (93) and a theorem of de la Vallee Poussin dealing with an equation (83) on a finite interval; [12], cf. [7]. 
Part III. The nonlinear case

7. $o(1)$-theorems. The consideration of this part will deal with the transfer of the results of Parts I and II from the linear system (2) to the nonlinear system

$$
y^{\prime}=J y+F(t, y),
$$

in which $F(t, y)$ is a continuous $d$-dimensional vector function of $(t, y)$ $=\left(t, y^{1}, \cdots, y^{d}\right)$ for large $t$ and small $|y|$. An analogue of the theorems (i) and (ii) on linear systems is the following theorem (in which the notation of (7), (9)-(13) is employed).

$\left(^{*}\right)$ Let the principal part $y^{\prime}=J y$ of (94) be the system (7). Let $\mu=\mu^{m}$ (in $(10)-(13))$ be negative. Let $F(t, y)$ be a continuous vector defined for large $t$ and small $|y|$. For some fixed $\theta>0$, let there exist a function $f(t)=f_{0}(t)$ satisfying, for large $t$,

$$
|F(t, y)| \leqq f(t)|y|
$$

on the $y$-set

$$
e^{\mu t} t^{-\theta} \leqq|y| \leqq e^{\mu t} t^{h_{*}-1+\theta}
$$

and, as $t \rightarrow \infty$,

$$
t^{h_{*} f(t) \rightarrow 0}
$$

or, more generally,

$$
(1+\log V)^{-1} \int_{U}^{U V} t^{h_{*}-1} f(t) d t \rightarrow 0 \text { when } \quad U V>U \rightarrow \infty .
$$

Then, if $T$ is sufficiently large there exists an $\left\{\sum_{p} h(p)+\sum_{(0)}(h(q))-b(q)\right.$ $+1)$ \}-dimensional set $\Omega=\Omega_{T}$ in the $y$-space such that if $y_{0}$ is a point of $\Omega$, then (94) has at least one solution $y=y(t)$ on $T \leqq t<\infty$ satisfying the initial condition $y(T)=y_{0}$ and the asymptotic relations $\left(15_{1}\right)-\left(15_{4}\right)$ as $t \rightarrow \infty$.

A partial converse of $(*)$ is given by:

(*bis) Let $J, \mu, F(t, y)$ satisfy the conditions of $(*)$, with the strengthened requirement that the inequality (95) holds on the $(t, y)$-set

$$
e^{(\mu-\theta) t} \leqq|y| \leqq e^{(\mu+\theta) t}
$$

(which contains (96)). Let $y=y(t)$ be a solution of (94) satisfying (96 bis) for large $t$ (for example, satisfying $(4)$ as $t \rightarrow \infty)$. Then $\left(17_{1}\right)-\left(17_{3}\right)$ and the relation $\left(17_{4}\right)$, for some integer $j_{0}\left(0 \leqq j_{0} \leqq h_{*}-1\right)$, hold. [6].

As to the existence of solutions $y=y(t)$ of (94) satisfying (4), see Part I in

Note that in the conservative case, where $F(t, y)=F(y)$, the conditions (95)-(96 bis), (97) are satisfied if 


$$
(-\log |y|)^{h_{*}}|F(y)| /|y| \rightarrow 0 \quad \text { as } \quad y \rightarrow 0
$$

(since $-\log |y| \geqq$ Const. $t$ as $t \rightarrow \infty$, when (96 bis) holds).

Consider the change (19) of independent variables and change of dependent variables $y \rightarrow z$ given by

$$
\begin{aligned}
& y^{q k}=e^{\lambda(q) t} t^{k-b(q)} \sum_{n=1}^{k} z^{q n} /(k-n) !, \\
& y^{j k}=e^{\mu t} t^{1-b} z^{j k} \text { if } j \neq q .
\end{aligned}
$$

Note that (99) is the variation of constants used in the proof of (ii); it combines the change of variables which accomplishes the reduction (18) and the change (20)-(22). Let (99) be denoted by $y=Q z$. Then (94) becomes

$$
\dot{z}=K(s) z+t Q^{-1} F(t, Q z),
$$$$
t=e^{s},
$$

where the principal part, $K(s) z$, is the same as in the system (23).

After this change of variables, assertion $\left({ }^{*}\right)$ above can be deduced from (*bis) and the Remark in $\$ 10,[6]$, as (ii) above was deduced from (III), $[5$, p. 61$]$. This deduction involves a detour, however, along the lines followed at the beginning of $\$ 19,[6]$. Note that if $z=z(s)$ is a solution of $(100)$ satisfying

$$
\begin{gathered}
s^{-e} \leqq|D| \leqq s^{\epsilon}, \\
\left|z^{j k}\right| \leqq \epsilon D \text { if }(j k) \neq(q b(q))
\end{gathered}
$$

on some $s$-interval, where $D=D(s)$ is defined in $\left(26_{1}\right)$, then the corresponding solution $y=y(t)$ satisfies inequalities of the type (96) if $\epsilon=\epsilon(\theta)>0$.

Write (94) as a linear system (2), in which the matrix $G(t)=G(t, y)$ depends on $y$ and is defined by

$$
G=\left(g_{j k}\right)=\left(F^{i}(t, y) \bar{y}^{k} /|y|^{2}\right),
$$$$
y \neq 0,
$$

where $F^{1}, \cdots, F^{d}$ are the components of $F$. Thus, if $z=z(s)$ is a solution of (100) on some $s$-interval satisfying (101) and $y=y(t)$ is the corresponding solution of (94), then (95)-(96) imply $\left|g_{j k}(t, y(t))\right| \leqq f(t)$. The change of variables (19) and $y=Q z$, given by (99), transforms (94) into (100) which can be written as a linear system, say (40), in which the matrix $H=H(s, z)$ is given by (24) and (102). Correspondingly, it follows that $|H(s, z)| \leqq$ Const. $t^{h} f(t)$ when $t=e^{s}$ and $z$ satisfies (101).

A comparison of (40) and (100) shows that $|H z|=t\left|Q^{-1} F(t, Q z)\right|$. Hence, the perturbation in (100) satisfies

$$
t\left|Q^{-1} F(t, Q z)\right| \leqq \text { Const. } t^{h_{*}} f(t)|z| \text { when (101) holds. }
$$

It follows from the deduction of $\left({ }^{*}\right)$, just sketched, that solutions $z=z(s)$ of (100) leading to solutions $y=y(t)$ of (94) satisfying (15) can result by an 
assignment of the initial values $z^{p k}(S), 1 \leqq k \leqq h(p)$, and $z^{q k}(S), b(q) \leqq k \leqq h(q)$, for sufficiently large $S$, where the $\left(\sum_{p} h(p)+\sum_{(0)}(h(q)-b(q)+1)\right)$ numbers $z^{p k}(S), z^{q k}(S)$ are subject to certain inequalities, but are otherwise arbitrary.

(*bis) above is a consequence of assertion ( $\mathrm{i}$ bis) in $\$ 1$, since (94) can be written as a linear system (2) in which $G(t)=G(t, y)$ is given by (102).

8. $O(1)$-variants. It is possible to obtain an analogue of $\left(I^{*}\right)$ for the nonlinear system (94) when the principal part (1) of (94) reduces to (28). To this end, the analogue of Lemma 1 will be indicated for the case where (40) is replaced by a nonlinear system

$$
\dot{z}=K(s) z+B(s, z) .
$$

LEMMA 2. Let $K=K(s)$ be a continuous (diagonal) $d$ by $d$ matrix on $0 \leqq s<\infty$ satisfying the conditions of Lemma 1 . Let $\mu$ and $\nu$, defined in (33), be constants. Let $B(s, z)$ be a continuous vector function of $(s, z)$ for large $s$ and $z$ satisfying

$$
\begin{aligned}
\exp \left(\mu-\theta c / 2(1-\epsilon)^{1 / 2}\right) s & \leqq|z| \leqq \exp \left(\nu+\theta c / 2(1-\epsilon)^{1 / 2}\right) s, \\
(M+N) & \leqq \epsilon L /(1-\epsilon)
\end{aligned}
$$

and let

$$
|B(s, z)| \leqq \theta c|z| / 2
$$

hold. Let $z_{0}^{j}$, where $j=p$ or $j=q$, be numbers satisfying

$$
\begin{gathered}
\sum_{p}\left|z_{0}^{p}\right|^{2} \leqq \xi \sum_{q}\left|z_{0}^{q}\right|^{2} \\
\exp \left(2 \mu-\theta c /(1-\epsilon)^{1 / 2}\right) S<\sum_{q}\left|z_{0}^{q}\right|^{2} \\
<(1-\epsilon) \exp \left(2 \nu+\theta c /(1-\epsilon)^{1 / 2}\right) S .
\end{gathered}
$$

Then, if $S>0$ is sufficiently large and $\xi>0$ is sufficiently small, there exists at least one solution $z=z(s)$ of (104) on $S \leqq s<\infty$ satisfying the (partial) set of initial conditions (41)-(42) and the asymptotic formulae (43), (44) as $s \rightarrow \infty$.

The proof of Lemma 2 will be omitted. It involves a careful rewriting of the proof of $(*),[6, \S 2-8]$. This procedure shows that condition (106) can be replaced by the inequalities

$$
g_{k}(s) \leqq \theta c / 2, \quad k=1,2,3,
$$

where

$$
\begin{aligned}
& \left(109_{1}\right) \quad g_{1}(s)=\max 2 \operatorname{Re}\left\{(L+N) \sum_{p} B^{p} \bar{z}^{p}-M \sum_{j=q, r} B^{j} \bar{z}^{j}\right\} /|z|^{4}, \\
& \left(109_{2}\right) \quad g_{2}(s)=\max 2 \operatorname{Re}\left\{N \sum_{j=p, q} B^{j} \bar{z}^{j}-(L+M) \sum_{r} B^{r} \bar{z}^{r}\right\} /|z|^{4},
\end{aligned}
$$




$$
g_{3}(s)=\max 2 \operatorname{Re}\left\{\sum_{q} B^{q} \bar{z}^{q}\right\} /|z|^{2},
$$

where $B^{i}=B^{j}(s, z)$ is the $j$ th component of $B=B(s, z)$, the $\max$ in $\left(109_{8}\right)$ refers to the maximum with respect to $z$ on the set (105) and, in (1091), $\left(109_{2}\right)$, to the common part of the set (105) and the respective sets

$$
\begin{aligned}
1-\epsilon / 2 & \leqq(L+N) /(M+L+N) \leqq 1-\delta, \\
\delta & \leqq N /(M+L+N) \leqq \epsilon / 2 .
\end{aligned}
$$

If the principal part (1) of (94) reduces to (28), the change of variables $t \rightarrow s=\log t$ and (65) transform (94) into (104), where

$$
B(s, z)=t Q^{-1}(t) F(t, Q z),
$$$$
t=e^{s},
$$

and $K=K(s)$ is the constant diagonal matrix with diagonal elements $\lambda^{1}=0$, $\lambda^{1}=-1, \cdots, \lambda^{d}=1-d$. Hence, $K$ satisfies the conditions of Lemma 1 if $j_{1}=j_{2}=m, \mu=\nu=1-m$ and $c=1$. Thus, if (111) satisfies the corresponding conditions of Lemma 2 , an analogue of $\left(I^{*}\right)$ results.

Appendix. On a THEOREM OF LonN

Consider the real, binary case of (94) and suppose that $J$ has a double elementary divisor, say, that (94) is of the type

$$
x^{\prime}=-x+F^{1}(t, x, y), \quad y^{\prime}=-y+x+F^{2}(t, x, y),
$$

where $x, y$ are real scalars and $F=\left(F^{1}, F^{2}\right)$ is a continuous binary vector satisfying

$$
|F| /\left(x^{2}+y^{2}\right)^{1 / 2} \rightarrow 0 \text { as }(t, x, y) \rightarrow(\infty, 0,0) .
$$

Let $(x, y)=(x(t), y(t)) \not \equiv 0$ be a solution of (112) which is defined for large $t$ and satisfies $(x(t), y(t)) \rightarrow(0,0)$ as $t \rightarrow \infty$. Then, as pointed out in [4, p. 501], $(x(t), y(t))$ has the properties that, as $t \rightarrow \infty$,

$$
2^{-1} \log \left(x^{2}+y^{2}\right) \sim-t
$$

and that either

$$
x(t) / y(t) \rightarrow 0 \text { or } \operatorname{arc} \tan x(t) / y(t) \rightarrow-\infty .
$$

In (115), it is understood that $\operatorname{arc} \tan x(t) / y(t)$ is chosen as a continuous function. In the general case (or even in the conservative cases) of (112), the alternative in (115) can depend on the particular solution $(x(t), y(t))[2$, p. 123], but is independent of the choice of the solution in the linear cases of (112) $[4$, p. 499$]$. It is clear that if

$$
y(t) \neq 0 \text { for large } t,
$$

then the first alternative in (115) must hold for the given solution. 
In view of Kneser's theorem mentioned after (91), it is natural to ask whether, when $x, y, F^{1}, F^{2}$ are real, there is a unilateral inequality (involving $F^{1}, F^{2}$ ) which assures that (116) (hence, the first alternative in (115)) holds for every solution of (112) satisfying (114). In this direction, the following will be proved:

(†) Let $F^{1}, F^{2}$ be real-valued continuous functions defined for large $t$ and real small $x$ and $y$. In addition to (113), assume that

$$
4 t^{2}\left(x F^{2}-y F^{1}\right) /\left(x^{2}+y^{2}\right) \leqq \theta<1
$$

holds for large $t$ on $a(t, x, y)$-set

$$
|x|<\xi|y|, \quad e^{-(1+\xi) t}<|y|<e^{-(1-\xi) t} \quad(\xi=\text { const. }>0) .
$$

Then every nontrivial $(\not \equiv 0)$ solution $(x, y)=(x(t), y(t))$ of $(112)$, defined for large $t$ and tending to $(0,0)$, as $t \rightarrow \infty$, satisfies (114) and the first alternative in (115).

In the conservative case, (117)-(118) can be replaced by the requirement that

$$
\left(x F^{2}-y F^{1}\right) \log ^{2}\left(x^{2}+y^{2}\right) /\left(x^{2}+y^{2}\right) \leqq \theta<1
$$

on an $(x, y)$-set

$$
|x|<\xi|y|, \quad x^{2}+y^{2}<\xi^{2} \quad(\xi=\text { const. }>0),
$$

since (118) implies that the ratio $4 t^{2} / \log ^{2}\left(x^{2}+y^{2}\right)$ is between $(1-\xi)^{-2}+o(1)$ and $(1+\xi)^{-2}$ as $t \rightarrow \infty$.

If $(112)$ is conservative and (117)-(118) is replaced by (119)-(120), then $(\dagger)$ reduces to a theorem of Lonn [8, p. 234]. Lonn also showed (loc. cit., p. 235) that if (119) is replaced by

$$
\left(x F^{1}-y F^{2}\right) \log ^{2}\left(x^{2}+y^{2}\right) /\left(x^{2}+y^{2}\right) \geqq C>4,
$$

then every solution tending to the origin satisfies the second alternative in (115). This statement of Lonn will be improved by relaxing the condition $C>4$ to $C>1$ (and will be made applicable to non-conservative cases).

$(\dagger \dagger)$ Let the assumptions of $(\dagger)$ hold except that $(117)-(118)$ is replaced by the requirement that

$$
4 t^{2}\left(x F^{2}-y F^{1}\right) /\left(x^{2}+y^{2}\right) \geqq C>1
$$

holds in (118). Then every solution $(x, y)=(x(t), y(t))$ of (112) defined for large $t$ and tending to the origin as $t \rightarrow \infty$ satisfies (114) and the second alternative in (115).

The proof of $(\dagger)$ which is similar to the procedure of Lonn will be given only for the sake of completeness. Actually, $(\dagger)$ and $(\dagger \dagger)$ depend essentially on the criteria, $4 t^{2} f(t) \leqq 1$ or $4 t^{2} f(t) \geqq C>1$, of Kneser for (91) to be non-oscillatory or oscillatory, respectively. 
Proof of $(\dagger)$. For large $t$, let $(x, y)=(x(t), y(t)) \rightarrow 0$ be a solution of (112) satisfying $(x, y) \rightarrow(0,0)$ as $t \rightarrow \infty$. Then (113) implies (114) and (115). If $y(t) \neq 0$ on some $t$-interval, then (112) shows that, on this $t$-interval, the function

$$
r=x / y
$$

satisfies the Riccati equation

$$
r^{\prime}+r^{2}+f(t)=0
$$

where

$$
f(t)=\left(x F^{2}-y F^{1}\right) / y^{2} \quad(x=x(t), y=y(t)) .
$$

Clearly, $1 \leqq\left(x^{2}+y^{2}\right) / y^{2} \leqq 1+\xi^{2}$ on the set (118). In what follows, it will be supposed that $\xi$ is a fixed positive number satisfying

$$
0<\left(1+\xi^{2}\right) \theta<1 \text {. }
$$

Hence, if $(t, x, y)=(t, x(t), y(t))$ is on the set (118), then (117) and (125) show that

$$
4 t^{2} f(t) \leqq\left(1+\xi^{2}\right) \theta<1 .
$$

The Riccati equation

$$
r^{\prime}+r^{2}+\left(1+\xi^{2}\right) \theta / 4 t^{2}=0
$$

has solutions of the form $r=\alpha / t$, where $\alpha$ satisfies the quadratic equation $-\alpha+\alpha^{2}+\left(1+\xi^{2}\right) \theta / 4=0$. In view of (125), the latter has (real) positive roots.

If (116) does not hold, then the second alternative in (115) holds. Hence (114) implies that, for some arbitrarily large $t$-values, $(t, x, y)=(t, x(t), y(t))$ satisfies (118). In fact, there exist arbitrarily large values, $t=t_{0}$ for which $x\left(t_{0}\right)$ $=\xi y\left(t_{0}\right) / 2>0$ holds and $(t, x, y)=\left(t_{0}, x\left(t_{0}\right), y\left(t_{0}\right)\right)$ satisfies (118). Then, if $t_{0}$ is sufficiently large, the function (122) satisfies $r\left(t_{0}\right)=\xi / 2>\alpha / t_{0}$. In view of (123) and (126), $r(t) \geqq \alpha / t>0$ on any interval $\left(t_{0}, t_{1}\right)$ on which (118), hence (126), holds. Since the second alternative in (115) holds there is a value (hence, a least value) of $t=t_{1}>t_{0}$, where $(t, x(t), y(t))$ is on the boundary of the set (118). If $t_{0}$ is sufficiently large, (114) and $r(t) \geqq \alpha / t>0$ imply that $x\left(t_{1}\right)=\xi y\left(t_{1}\right)>0$. Hence

$$
r\left(t_{1}\right)=\xi \text { and } 0<r(t)<\xi \text { if } t_{0} \leqq t<t_{1} .
$$

Thus (123) becomes $r^{\prime}\left(t_{1}\right)+\xi^{2}+f\left(t_{1}\right)=0$ at $t=t_{1}$. In view of (114) and $x\left(t_{1}\right)$ $=\xi y\left(t_{1}\right)$, the assumption (113) implies that $\left|f\left(t_{1}\right)\right|<\xi^{2}$ if $t_{0}$ (hence $\left.t_{1}\right)$ is suffciently large. Consequently, $r^{\prime}\left(t_{1}\right)<0$. Since this contradicts $(128)$, the assumption that (116) does not hold is untenable. This proves $(\dagger)$.

Proof of $(\dagger \dagger)$. Let $(x, y)=(x(t), y(t))$ be a solution of (112) for large $t$ tending to the origin as $t \rightarrow \infty$. Then (114) and (115) hold. Suppose, if possible, 
that the first alternative of (115) holds; in particular, that (116) holds. In view of (114) and the first part of (115), the point $(t, x, y)=(t, x(t), y(t))$ is on the set (118) for any fixed $\xi>0$ and sufficiently large $t$.

Since (116) holds, (122) defines a continuously differentiable function for large $t$ satisfying (123)-(124). The inequality (121) shows that $4 t^{2} f(t) \geqq C\left(x^{2}\right.$ $\left.+y^{2}\right) / y^{2} \geqq C>1$, and so $4 t^{2} f(t) \geqq C>1$ for large $t$. This implies that (91) is oscillatory (Kneser). Hence the Riccati equation (123), belonging to (91), cannot possess a solution $r=r(t)$ defined for large $t$. Thus, the assumption (116) leads to a contradiction, and so ( $\dagger$ ) follows.

\section{REFERENCES}

1. P. Hartman, Unrestricted solution fields of almost separable differential equations, Trans. Amer. Math. Soc. vol. 63 (1948) pp. 560-580.

2. P. Hartman and A. Wintner, On the behavior of the solutions of real binary differential systems at singular points, Amer. J. Math. vol. 75 (1953) pp. 117-126.

3. - On non-oscillatory differential equations, ibid. vol. 75 (1953) pp. 717-730.

4. - On curves defined by binary non-conservative differential systems, ibid. vol. 76 (1954) pp. 497-501.

5. - Asymptotic integrations of linear differential equations, ibid. vol. 77 (1955) pp. 4586 and p. 932.

6. - Asymptotic integrations of ordinary non-linear differential equations, ibid. vol. 77 (1955) pp. 692-724.

7. - On an oscillation criterion of de la Vallée Poussin, Quarterly of Applied Mathematics vol. 13 (1955) pp. 330-332.

8. E. Lonn, Knoteninvarianz bei Differentialgleichungen, Jber. Deutschen Math. Verein. vol. 43 (1934) pp. 232-237.

9. O. Perron, Über Stabilität und asymptotisches Verhalten der Integrale von Differentialgleichungssystemen, Math. Zeit. vol. 29 (1929) pp. 129-160.

10. - Die Stabilitätsfrage bei Differentialgleichungen, ibid. vol. 32 (1930) pp. 703-728.

11. Z. Szmydtowna, Sur l'allure asymptotique des intégrales des équations différentielles ordinaires, Annales de la Société Polonaise de Mathématique vol. 24 (1951) pp. 17-34.

12. C. de la Vallée Poussin, Sur l'équation différentielle linéaire du second ordre. Détermination d'une intégrale par deux valeurs assignées. Extension aux équations d'ordre n, J. Math. Pures Appl. vol. 8 (1929) pp. 125-144.

The Johns Hopkins University,

BALTimore, MD. 